\title{
Use of Atrial Fibrillation Organization to Evaluate the Efficacy of Guided Ablation
}

\author{
Carlos Roberto ${ }^{1}$, Laura Martínez-Mateu ${ }^{2}$, Ana Andrés ${ }^{1}$, Joaquín Osca ${ }^{1}$, Javier Saiz ${ }^{2}$ \\ ${ }^{1}$ Hospital Universitario y Politécnico La Fe, Valencia, Spain \\ ${ }^{2}$ Centro de Investigación e Innovación en Bioingeniería (Ci2B), Universitat Politècnica de València, \\ Valencia, Spain
}

\begin{abstract}
Catheter ablation is a widely-used therapy to treat atrial fibrillation $(A F)$, but the identification of ablation targets remain challenging in persistent AF (PersAF). This work aims to study the anatomical relationship between rotors and areas of low-voltage, in addition to evaluate the outcomes of PersAF ablation guided by rotors maps in combination with pulmonary vein isolation, through frequency analysis of the electrograms (EGMs).

Eight patients with PersAF and no previous ablation procedure were included in this study. Ablation procedures were guided by Carto $^{\circledR} 3$ voltage maps and CardioInsight ${ }^{T M}$ rotors maps. The location of rotors was qualitatively compared with low-voltage areas $(<0.5 \mathrm{mV})$. In addition, frequency analysis was carried out with five bipolar EGMs, recorded by using a decapolar catheter located in the coronary sinus. Spectral analysis was performed using the Welch method. The highest peak in the spectrum was defined as the dominant frequency (DF). To measure the periodicity and organization of the signals, the regularity (IR) and organization (IO) indexes were implemented.

Our results showed that rotors location largely corresponded to low-voltage zones (85.7\%). In addition, although only one patient restored sinus rhythm after ablation and the remaining patients needed cardioversion, an improvement in DF, RI and OI was observed in $75 \%$ of cases.
\end{abstract}

\section{Introduction}

Atrial fibrillation (AF) is the most common arrhythmia in clinical practice, affecting 1-2 \% of the general population. The loss of effective atrial contraction in AF patients can result in blood clots in the atria, which increases the risk of stroke five-fold [1].

Catheter ablation is a widely-used therapy and treats $\mathrm{AF}$ by either eliminating the trigger or altering the arrhythmogenic substrate [2]. Treating paroxysmal AF with pulmonary veins isolation (PVI) in clinical practice is based on evidence that triggered firing originates from pulmonary veins (PV) $[3,4]$, but the poor results in treating persistent AF (PersAF) patients with the same ablation protocol points to the importance of AF drivers outside the pulmonary veins [5], with the underlying atrial substrate being either structural or electrical [6].

For the study of the spatiotemporal organization of AF, different signal analysis techniques are currently being used to analyze the electrograms (EGMs) recorded at different points of the atria. These include the analysis of EGMs morphology [7], dominant frequencies (DF) [8] and regularity (RI) [9] or organization indexes (OI) [10]. Evidence from high-density mapping and spectral analysis has shown that AF is associated with rotors and regular repetitive activation in a region of the atria. In addition, areas of high DF have been proposed as critical regions for maintaining AF [11]. Thus, identification of areas with these features may allow targeted ablation procedures to improve success rates [12].

This work aims to study the anatomical relationship between rotors and areas of low-voltage, in addition to evaluate the outcomes of PersAF ablation guided by rotors maps in combination with PVI, through the analysis of intracavitary EGMs. For this purpose, a frequency analysis will be performed, including DF, RI and OI calculation.

\section{Materials and Methods}

Eight patients with PersAF and no previous catheter ablation procedure were included in the study. Baseline characteristics of patients are presented in Table 1. Antiarrhythmic agents were not discontinued and patients maintained their anticoagulant treatment.

\subsection{Ablation Procedures}

Ablation procedures, performed under general anesthesia, were guided by $\mathrm{Carto}^{\circledR} 3$ (Biosense Webster, Inc.) and CardioInsight ${ }^{\mathrm{TM}}$ (Medtronic, Minneapolis, MN) mapping systems. Before ablation, both voltage maps 
provided by Carto $^{\circledR} 3$ and rotors maps provided by CardioInsight $^{\mathrm{TM}}$ were built. A 20-pole catheter (Pentaray 2-6-2 mm F curve 7 Fr, Biosense-Webster, Diamond Bar, CA) was used for atrial mapping (Carto ${ }^{\mathbb{B}} 3$ voltage maps) and a body surface potential mapping vest of 252 electrodes was employed for CardioInsight ${ }^{\mathrm{TM}}$ rotors maps. In addition, a 4-mm irrigated-tip ablation catheter (SmartTouch SF F curve 7'5 Fr, Biosense-Webster, Diamond Bar, CA) was used for PVI and substrate ablation, and a 6Fr decapolar catheter (Webster CS Catheter, Biosense-Webster, Diamond Bar, CA) was positioned in the coronary sinus (CS) as a reference.

Once voltage and rotors maps were completed, ablation was performed in two steps: first, PV were isolated and then, according to the rotors map, areas with rotational activity were ablated. Substrate ablation was guided by rotor maps only for those cases in which the information provided by CardioInsight ${ }^{\mathrm{TM}}$ and Carto ${ }^{\circledR} 3$ agreed, i.e. when rotor areas in the rotors maps coincided with low-voltage areas $(<0$ '5 $\mathrm{mV})$ in the voltage maps. Therefore, the location of each rotors and low-voltage areas were analysed to determine their correspondence. For the remaining cases, substrate ablation was only guided by Carto $^{\circledR} 3$ and the low-voltage areas. All the procedures were performed by the same operator.

Table 1. Baseline Characteristics of Patients. OSAS: Obstructive Sleep Apnea Syndrome; LA: Left Atrium; AF: Atrial Fibrillation.

\begin{tabular}{ll}
\hline Age (years) & $63,125 \pm 8$ \\
Female & $2(25 \%)$ \\
Structural Heart disease & $3\left(377^{\prime} \%\right)$ \\
Hypertension & $\left.5(62)^{\prime} \%\right)$ \\
Dyslipidaemia & $4(50 \%)$ \\
Diabetes Mellitus & $\left.1(12)^{\prime} 5 \%\right)$ \\
OSAS & $1(12,5 \%)$ \\
Smoking & $2(25 \%)$ \\
LA enlargement & $5(62,5 \%)$ \\
AF duration (years) & $5,4 \pm 5,5$ \\
\hline
\end{tabular}

\subsection{Data Acquisition and Frequency Analysis}

Bipolar EGMs from the reference catheter in the CS were exported from a digital amplifier/recording system (Bard Electrophysiology, Lowell, MA) to Matlab ${ }^{\circledR}$ (MathWorks, Natick, Massachusetts, USA) at a sampling rate of $1 \mathrm{kHz}$ and 1 -minute duration. These recordings were bandpass filtered $(30-500 \mathrm{~Hz})$, rectified and lowpass filtered $(20 \mathrm{~Hz})[13,14,15]$.

Once signals were preprocessed, frequency analysis was performed as follows. First, power spectral density was estimated via Welch's method over a sliding 4-second window (Hamming window) with $50 \%$ overlapping. Zero- padding was applied resulting in a frequency resolution of $0.0167 \mathrm{~Hz}$. Then, the highest peak of the spectrum was identified and defined as the DF. The spectral power of the DF and its first three harmonics was calculated as the area under their peaks over a $1 \mathrm{~Hz}$ window, whereas the total power of the spectrum was calculated as the area under the spectrum between $0.5 \mathrm{~Hz}$ and $15 \mathrm{~Hz}$, since this frequency range is considered to be physiological [16]. Finally, in order to measure the periodicity of the signal and the variability of frequency in the spectrum, the RI and OI indexes were implemented. The RI was defined as the ratio of the DF spectral power to the total power of the spectrum, while the OI was defined as the ratio of the spectral power of the DF together with its first three harmonics to the total power of the spectrum.

This frequency analysis was performed for 1-minute recordings corresponding to different phases of the ablation procedure: basal EGM prior to ablation, EGM recorded after each region ablation and EGM recorded at the end of the procedure.

\section{Results and discussion}

At the end of the ablation procedure only 1 patient recovered sinus rhythm (SR) (Patient 1). During the ablation procedure another patient was led to atrial flutter and then restored SR. However, due to stimulation manoeuvres, this patient (Patient 2) fell again into AF. Rest of patients were cardioverted to SR after ablation procedure. No recurrence of AF has been observed in any patient ( 6 of them have exceeded the 3 -month period of post-ablation blanking, with an average follow-up time of 9 months). Nevertheless, $33 \%$ of patients have shown recurrence as atrial flutter.

\subsection{Anatomical matching of rotors and low-voltage areas}

A total number of 53 low-voltage areas and 52 rotor regions were visually detected on the maps. The number of low-voltage regions found in the left atrium (LA) ascended to 47 , while in the right atrium (RA) was 6 . Carto $^{\circledR} 3$ electroanatomical maps were built either for both atria or only the LA, depending on the physician criterion (RA was only mapped in the $37.5 \%$ of cases (3 patients)). However, rotors maps were always comprised of LA and RA, being the distribution 36 rotor regions in the LA versus 6 in the RA. Among the 52 rotor regions detected, only 42 were evaluable due to the lack of RA voltage map.

In order to qualitatively compare the location of the detected rotors and low-voltage areas, the atrial anatomy was divided into 10 regions: anterior wall, posterior wall, roof, septum, left pulmonary veins, right pulmonary veins, left appendage, right appendage, left carina and right carina. Then, different views of both type of maps were 
taken: anterior-posterior, posterior-anterior, left oblique, right oblique, superior and inferior. As a result, we observed that the location of $85.7 \%$ of the rotors regions corresponded to low-voltage areas. The regions with a higher number of rotors detection was the posterior wall of the LA and the PV ( $87.5 \%$ of patients) followed by the roof and septum of the LA ( $75 \%$ of patients). In $37.5 \%$ of patients, all the detected rotors presented a corresponding low-voltage area, so in those cases substrate ablation was guided by CardioInsight ${ }^{\mathrm{TM}}$. This means that in the $62.5 \%$ of patients $\operatorname{Carto}^{\mathbb{B}} 3$ was also needed to ablate the substrate. Figure 1 shows rotors (left) and voltage (right) maps from patient 8 . As observed, in this case both maps matched perfectly.
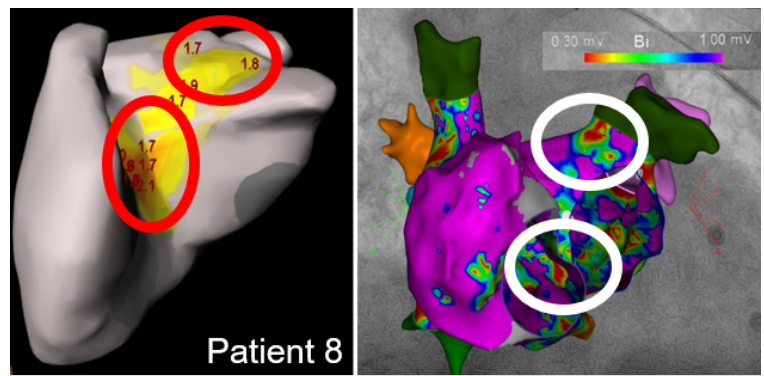

Figure 1. Comparison of rotors (left) and voltage (right) maps from patient 8 (anterior-posterior view). Yellow to orange areas on the rotors map indicate the presence of rotors (number of cycles of each rotor superimposed). Red regions on the voltage maps correspond to low-voltage areas.

Frequency domain analysis was performed for the five bipolar EGMs from the reference catheter positioned in the $\mathrm{CS}$, at a stable location to allow unbiased measurements. Among all the EGMs, the one showing no or minimal ventricular activity was chosen to analyse the pre- to postablation frequency variation. In the majority of patients (75\%) that EGM was the proximal recording. Although only one patient returned to SR during ablation (12.5\%), the ablation procedures did lead to an improvement in the spectral characteristics of the EGMs. DF decreased (slowdown of the AF) and both the OI and the RI indexes increased (EGMs became more regular and organized) after ablation for 6 of the 8 patients $(75 \%)$. A summary of the ablation outcomes is shown in Table 2. In addition, Figure 2 depicts the EGMs, and their corresponding spectra, prior and after the ablation procedure for patient 1 (A) and 3 (B). Patient 1 recovered SR after ablation, as easily observed in panel A. DF was reduced from $5.317 \mathrm{~Hz}$ to $1.033 \mathrm{~Hz}$, and RI and OI increased from 0.192 and 0.300 to 0.779 and 0.926 , respectively. Unlike patient 1 , patient 3 remained in AF after ablation and there was not a strong improvement in DF. However, RI and OI did improve (from 0.196 and 0.268 to 0.301 and 0.422 ).

\section{A) Patient 1}
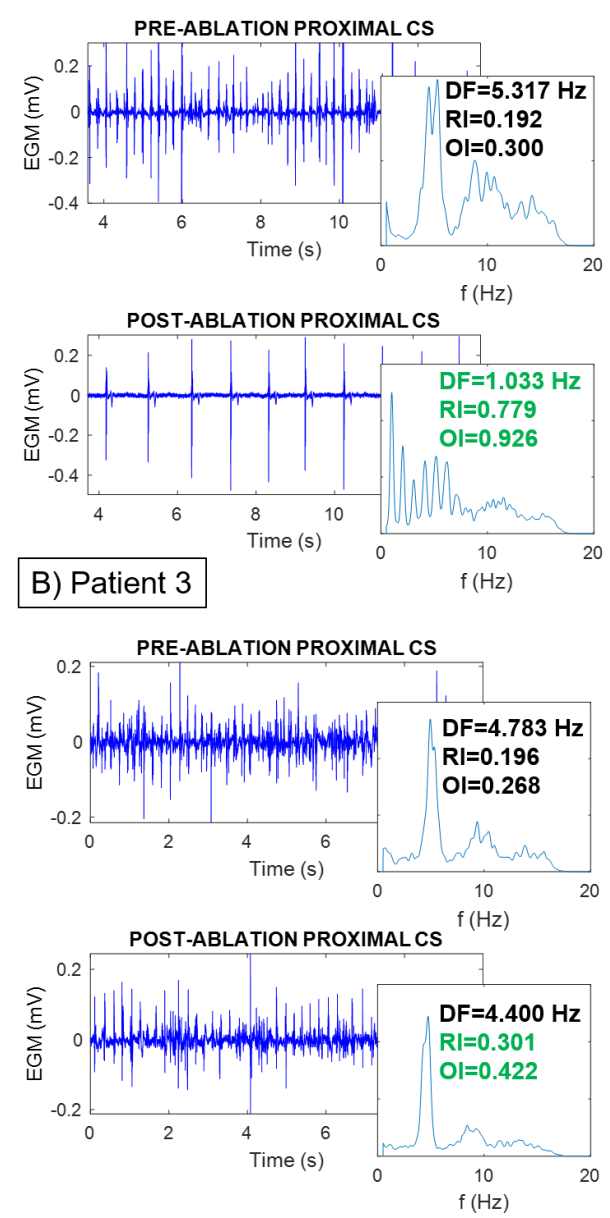

Figure 2. EGMs and power spectra corresponding to patient 1 (A) and $3(B)$.

Table 2. DF, RI and OI variations between pre-ablation and post-ablation recordings.

\begin{tabular}{|l|c|c|c|c|c|c|}
\hline & \multicolumn{3}{|c|}{ Pre-ablation } & \multicolumn{3}{c|}{ Post-ablation } \\
\cline { 2 - 7 } & DF & RI & OI & DF & RI & OI \\
\hline Patient1 & 5,317 & 0,192 & 0,300 & 1,033 & 0,779 & 0,926 \\
\hline Patient2 & 4,167 & 0,310 & 0,456 & 4,367 & 0,363 & 0,631 \\
\hline Patient3 & 4,783 & 0,196 & 0,268 & 4,400 & 0,301 & 0,422 \\
\hline Patient4 & 4,633 & 0,289 & 0,462 & 5,617 & 0,283 & 0,384 \\
\hline Patient5 & 6,317 & 0,350 & 0,457 & 5,783 & 0,514 & 0,597 \\
\hline Patient6 & 5,033 & 0,325 & 0,440 & 4,650 & 0,418 & 0,573 \\
\hline Patient7 & 6,467 & 0,382 & 0,432 & 5,550 & 0,188 & 0,275 \\
\hline Patient8 & 7,250 & 0,207 & 0,254 & 6,317 & 0,250 & 0,292 \\
\hline
\end{tabular}

Similarly to previous studies in which patients under PersAF conditions were mapped with CardioInsight ${ }^{\mathrm{TM}}[17$, $18]$, all 8 patients in this work presented rotational activity 
and PV were within the most frequent rotors locations. Moreover, although the correlation between rotors and low-voltage areas was similar to that one previously observed between rotors and complex fractionated atrial EGMs (CFAEs) [18], in our work ablation success was much lower (only patient 1 recovered SR without cardioversion). Nevertheless, the increase of RI and OI in $75 \%$ of patients suggested less variability of frequency, which implies less drivers' activity in the atria [13].

\section{Conclusions}

Our study showed that CardioInsight ${ }^{\mathrm{TM}}$ rotor locations largely corresponded to low-voltage areas in Carto ${ }^{\circledR} 3$ electroanatomical maps (85.7\%). However, only in $37.5 \%$ of patients all the detected rotors corresponded to a lowvoltage area. In addition, although $87.5 \%$ of patients had to be cardioverted to SR after the ablation procedure, DF, RI and OI improved in $75 \%$ of cases. It should be noted that the rrelationship between these three parameters (DF, $\mathrm{RI}$ and $\mathrm{OI}$ ) and the recovery of SR during ablation remains unclear and that the low number of patients enrolled might be a potential limitation of this work. Therefore, there is a need of further studies for a better understanding of the EGMs spectral characteristics leading to a successful ablation.

\section{Acknowledgments}

This work was supported by Programa Prometeu de la Conselleria d'Educació, Formació I Ocupació de la Generalitat Valenciana, award number PROMETEU 2016/088; Plan Estatal de Investigación Científica y Técnica y de Innovación 2013-2016 del Ministerio de Economía, Industria y Competitividad of Spain, Agencia Estatal de Investigación and the European Commission (European Regional Development Funds ERDF-FEDER), award number DPI201675799-R; and the Hospital Universitario y Politécnico La Fe.

\section{References}

[1] G. Lip et al., "Atrial fibrillation", Nature Reviews Disease Primers, vol. 2, no. 1, 2016.

[2] M. Haïssagurrre, F. Marcus, B. Fischer and J. Clémenty, "Radiofrequency Catheter Ablation in Unusual Mechanisms of Atrial Fibrillation: report of three cases." JCardiovasc Electrophysiol, vol. 5, no. 9, pp. 743-751, 1994.

[3] P. Jaïs et al., "A Focal Source of Atrial Fibrillation Treated by Discrete Radiofrequency Ablation", Circulation, vol. 95, no. 3, pp. 572-576, 1997.

[4] M. Haïssaguerre et al., "Spontaneous Initiation of Atrial Fibrillation by Ectopic Beats Originating in the Pulmonary Veins", New England Journal of Medicine, vol. 339, no. 10, pp. 659-666, 1998.

[5] J. Jalife, "Mother rotors and fibrillatory conduction: a mechanism of atrial fibrillation", Cardiovascular Research, vol. 54, no. 2, pp. 204-216, 2002.

[6] C. Morillo, G. Klein, D. Jones and C. Guiraudon, "Chronic Rapid Atrial Pacing", Circulation, vol. 91, no. 5, pp. 1588$1595,1995$.

[7] V. Jacquemet et al., "Study of Unipolar Electrogram Morphology in a Computer Model of Atrial Fibrillation", Journal of Cardiovascular Electrophysiology, vol. 14, no. 10, pp. S172-S179, 2003.

[8] Y. Lin, C. Tai and S. Chen, "Can Mapping and Ablation of Atrial Fibrillation Be Guided by Frequency Analysis of Fibrillatory Waves?" Journal of Cardiovascular Electrophysiology, vol. 17, no. 3, pp. S44-S49, 2006.

[9] P. Sanders, O. Berenfeld and M. Hocini, "Spectral Analysis Identifies Sites of High-Frequency Activity Maintaining Atrial Fibrillation in Humans", ACC Current Journal Review, vol. 14, no. 12, p. 40, 2005.

[10] T. Everett, Lai-Chow Kok, R. Vaughn, R. Moorman and D. Haines, "Frequency domain algorithm for quantifying atrial fibrillation organization to increase defibrillation efficacy", IEEE Transactions on Biomedical Engineering, vol. 48, no. 9, pp. 969-978, 2001.

[11] R. Mandapati, A. Skanes, J. Chen, O. Berenfeld and J. Jalife, "Stable Microreentrant Sources as a Mechanism of Atrial Fibrillation in the Isolated Sheep Heart", Circulation, vol. 101, no. 2, pp. 194-199, 2000.

[12] C. Tobón, C. Ruiz-Villa, E. Heidenreich, L. Romero, F. Hornero and J. Saiz, "A Three-Dimensional Human Atrial Model with Fiber Orientation. Electrograms and Arrhythmic Activation Patterns Relationship", PLoS ONE, vol. 8, no. 2, p. e50883, 2013

[13] Takahashi Y, Sanders P, Jais P et al. "Organization of Frequency Spectra of Atrial Fibrillation: Relevance to Radiofrequency Catheter Ablation." $J$ Cardiovasc Electrophysiol. 2006;17(4):382-388.

[14] T. Everett, E. Wilson and J. Olgin, "Effects of atrial fibrillation substrate and spatiotemporal organization on atrial defibrillation thresholds", Heart Rhythm, vol. 4, no. 8, pp. 1048-1056, 2007.

[15] J. Akar, T. Everett, L. Kok, J. Moorman and D. Haines, "Effect of Electrical and Structural Remodeling on Spatiotemporal Organization in Acute and Persistent Atrial Fibrillation", Journal of Cardiovascular Electrophysiology, vol. 13, no. 10, pp. 1027-1034, 2002.

[16] T. Everett, Lai-Chow Kok, R. Vaughn, R. Moorman and D. Haines, "Frequency domain algorithm for quantifying atrial fibrillation organization to increase defibrillation efficacy", IEEE Transactions on Biomedical Engineering, vol. 48, no. 9, pp. 969-978, 2001.

[17] M.P. Ehrlich e al., "Non invasive mapping before surgical ablation for persistent, long-standing atrial fibrillation", $J$ Thorac Cardiovasc Surg, vol. 157, no. 1, pp. 248-256, 2019

[18] S. Ammar-Busch et al., "Correlation between atrial fibrillation driver locations and complex fractionated atrial electrograms in patients with persistent atrial fibrillation", Pacing Clin Electrophysiol, vol.41, pp. 1279-1285, 2018

Address for correspondence:

Javier Saiz

Centro de Investigación e Innovación en Bioingeniería (Ci2B), Universitat Politècnica de València,

jsaiz@ci2b.upv.es 